\title{
2014 Annual Industrial
} Wastewater Reuse Report for the Idaho National Laboratory Site's Materials and Fuels Complex Industrial Waste Ditch and Industrial Waste Pond

Michael G. Lewis

February 2015

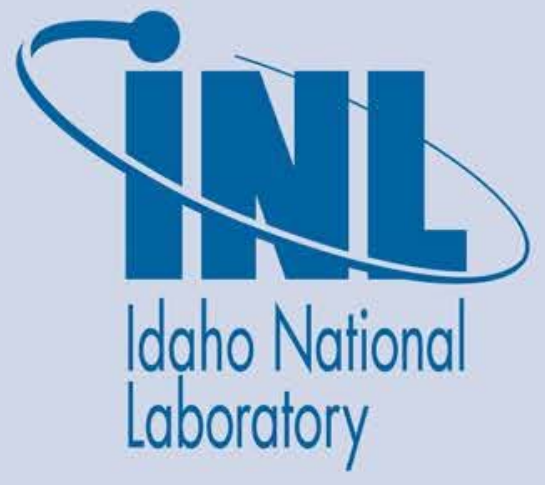

The INL is a U.S. Department of Energy National Laboratory operated by Battelle Energy Alliance 



\title{
2014 Annual Industrial Wastewater Reuse Report for the Idaho National Laboratory Site's Materials and Fuels Complex Industrial Waste Ditch and Industrial Waste Pond
}

\author{
Michael G. Lewis
}

February 2015

Idaho National Laboratory

Idaho Falls, Idaho 83415

http://www.inl.gov

Prepared for the

U.S. Department of Energy

Office of Nuclear Energy, Science, and Technology

Under DOE Idaho Operations Office

Contract DE-AC07-05ID14517 



\begin{abstract}
This report describes conditions, as required by the state of Idaho Industrial Wastewater Reuse Permit (WRU-I-0160-01, formerly LA-000160-01), for the wastewater reuse site at the Idaho National Laboratory Site's Materials and Fuels Complex Industrial Waste Ditch and Industrial Waste Pond from November 1, 2013 through October 31, 2014. The report contains the following information:
\end{abstract}

- Facility and system description

- Permit required effluent monitoring data and loading rates

- Groundwater monitoring data

- Status of special compliance conditions

- Noncompliance issues

- Discussion of the facility's environmental impacts

During the 2014 reporting year, an estimated 10.11 million gallons of wastewater were discharged to the Industrial Waste Ditch and Pond which is well below the permit limit of 17 million gallons per year. The concentrations of all permit-required analytes in the samples from the down gradient monitoring wells were below the applicable Idaho Department of Environmental Quality's groundwater quality standard levels. 


\section{CONTENTS}

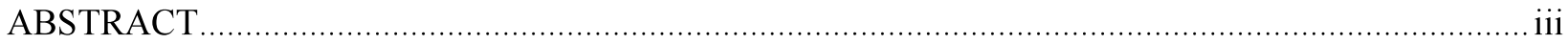

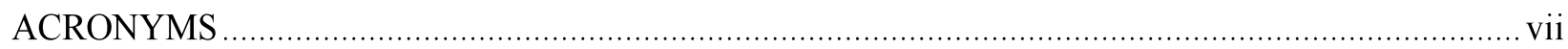

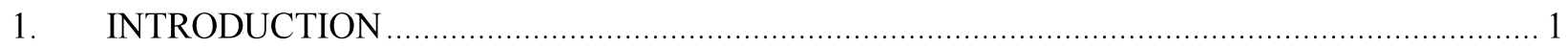

2. FACILITY, SYSTEM DESCRIPTION, AND OPERATION …............................................... 1

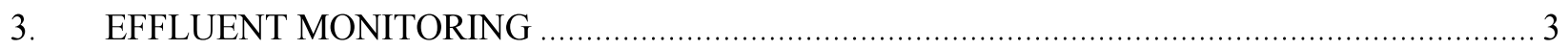

3.1 Sampling Program and Analytical Methods .................................................................. 3

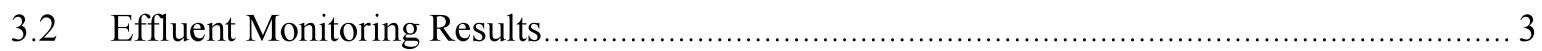

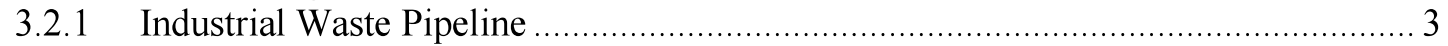

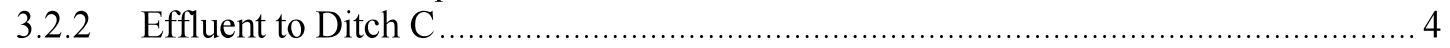

3.3 Flow Volumes and Hydraulic Loading Rates ....................................................................... 10

3.3.1 Industrial Waste Pipeline to Industrial Waste Pond .............................................. 10

3.3.2 Industrial Waste Water Underground Pipeline to Ditch C.................................... 10

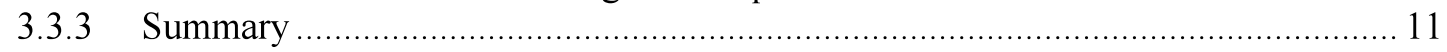

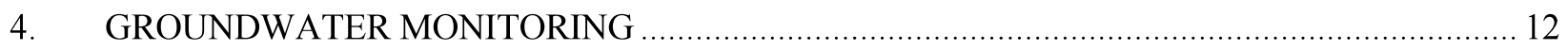

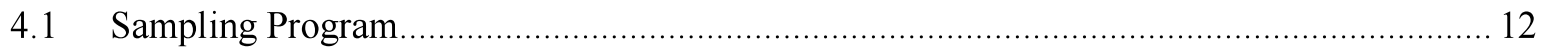

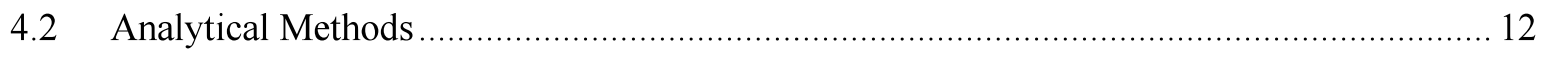

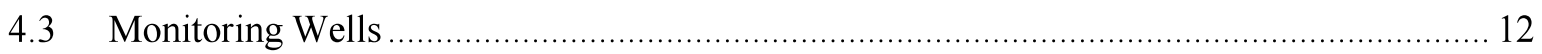

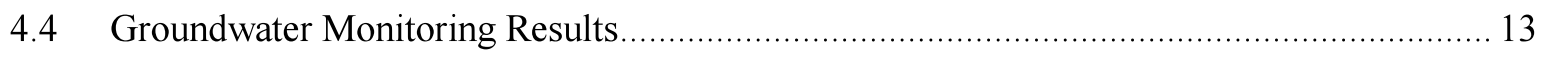

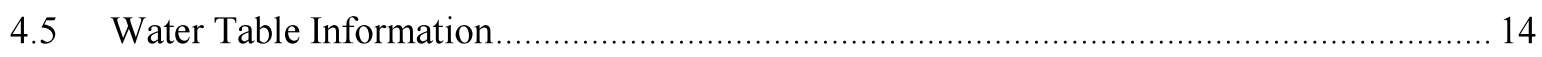

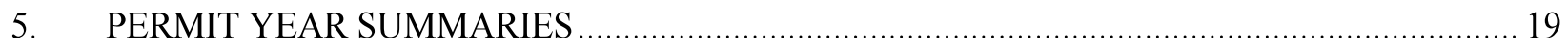

5.1 Status of Permit Required Compliance Activities............................................................ 19

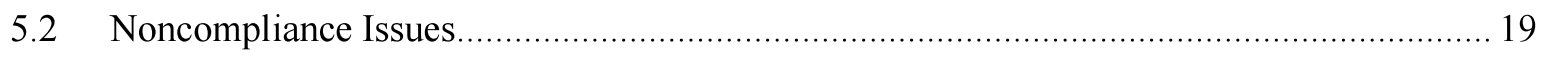

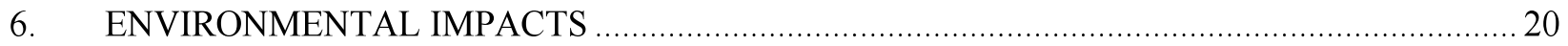

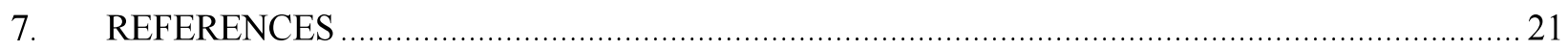

\section{FIGURES}

Figure 1. MFC Industrial Waste Ditch and Industrial Waste Pond. ................................................ 2

Figure 2. Iron, lead, and manganese concentrations in the Industrial Waste Pipeline from

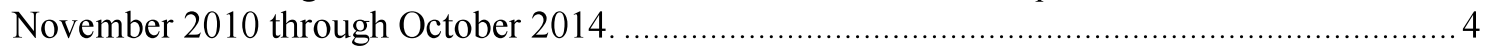

Figure 3. Iron concentrations in the unfiltered and filtered samples from the three aquifer monitoring wells for the period of May 2010 through September 2014 ............................. 13

Figure 4. Groundwater contour map based on the April/May 2014 water level measurements............... 17

Figure 5. Groundwater contour map based on the September 2014 water level measurements............... 18 
Figure 6. Total suspended solids concentrations in samples collected from Ditch C for the period of May 2010 through August 2014.

\section{TABLES}

Table 1. Analytical results for 24-hour composite samples collected from the Industrial Waste Pipeline (WW-016001)

Table 2. Analytical results for quarterly grab samples collected from the wastewater discharged to Ditch C from the Industrial Waste Water Underground Pipe (WW-016002).

Table 3. Volume of wastewater discharged from the Industrial Waste Pipeline to the Industrial Waste Pond.

Table 4. Volume of wastewater discharged from the Industrial Waste Water Underground Pipe to Ditch C

Table 5. Summary of groundwater quality data collected for the Wastewater Reuse Permit for the MFC Industrial Waste Ditch and Pond. 


\section{ACRONYMS}

BEA Battelle Energy Alliance, LLC

bgs below ground surface

CFR Code of Federal Regulations

DEQ Idaho Department of Environmental Quality

gpm gallons per minute

IDAPA Idaho Administrative Procedures Act

INL Idaho National Laboratory

IWD Industrial Waste Ditch

IWP Industrial Waste Pond

IWRP Industrial Wastewater Reuse Permit

MFC Materials and Fuels Complex

MG Million gallons

NA Not Applicable

PCS Primary Constituent Standard

R\&MS Regulatory and Monitoring Services

SCS Secondary Constituent Standard

TN total nitrogen

TSS total suspended solids 


\section{Annual Industrial Wastewater Reuse Report for the Idaho National Laboratory Site's Materials and Fuels Complex Industrial Waste Ditch and Industrial Waste Pond}

\section{INTRODUCTION}

The Materials and Fuels Complex (MFC) Industrial Waste Ditch (IWD) and associated Industrial Waste Pond (IWP) is an industrial wastewater reuse facility operated by Battelle Energy Alliance, LLC (BEA) under Industrial Wastewater Reuse Permit (IWRP) WRU-I-0160-01 (formerly LA-000160-01) issued by the Idaho Department of Environmental Quality (DEQ). The initial permit was issued in April 2010; Modification 1 was issued in June 2012 (Neher 2010; Neher 2012). The permit will expire on April 30, 2015 (Neher 2012).

As required by the IWRP, the permit renewal application is to be submitted six months prior to the IWRP expiration date. The renewal application, designation of Responsible Official and Authorized Representative form, and facility information form were submitted to DEQ on October 28, 2014 (Miller 2014a).

The annual report summarizes the facility system and operation, monitoring data, special compliance conditions, noncompliances, and environmental impacts for the 2014 reporting year (November 1, 2013 through October 31, 2014).

\section{FACILITY, SYSTEM DESCRIPTION, AND OPERATION}

The MFC is located on approximately 60 acres in the southeastern portion of the Idaho National Laboratory (INL), approximately 30 miles west of Idaho Falls, Idaho, in Bingham County. The MFC consists of buildings and structures for research and development on nuclear technologies, nuclear environmental management, and space radioactive power source development.

The IWP is located near the northwest corner of the MFC (Figure 1). The IWP was first excavated in 1959 and has a design capacity of 285 million gallons (MG) at a maximum water depth of 13 feet.

Industrial wastewater discharged to the IWP system consists primarily of noncontact cooling water, boiler blowdown, cooling tower overflow, air wash flows, and steam condensate. Small amounts of industrial wastewater from the MFC facility process holdup tanks may also be discharged to the IWP system, once approved by the facility supervisor and environmental compliance staff. The IWP also receives storm water runoff from MFC and immediate environs.

Most of the industrial wastewater generated at MFC flows through collection piping to a lift station where it is pumped into the Industrial Waste Pipeline and discharged to the pond (Figure 1). A flow meter and composite sampler are located on the pipeline near the western boundary of MFC (Figure 1, WW016001).

Wastewater composed of cooling water blowdown, intermittent reverse osmosis effluent, and discharge to floor drains and a laboratory sink is transported from the MFC-768 Power Plant to IWD (Ditch C) via the Industrial Waste Water Underground Pipe (Figure 1). The wastewater discharged to Ditch C seldom flows more than a few tens of feet past the sampling point (WW-016002) before it evaporates, infiltrates, or is taken up by plants.

The maximum hydraulic loading rate allowed by the permit for both discharges, IWP and Ditch C, is 17 million gallons/year. 


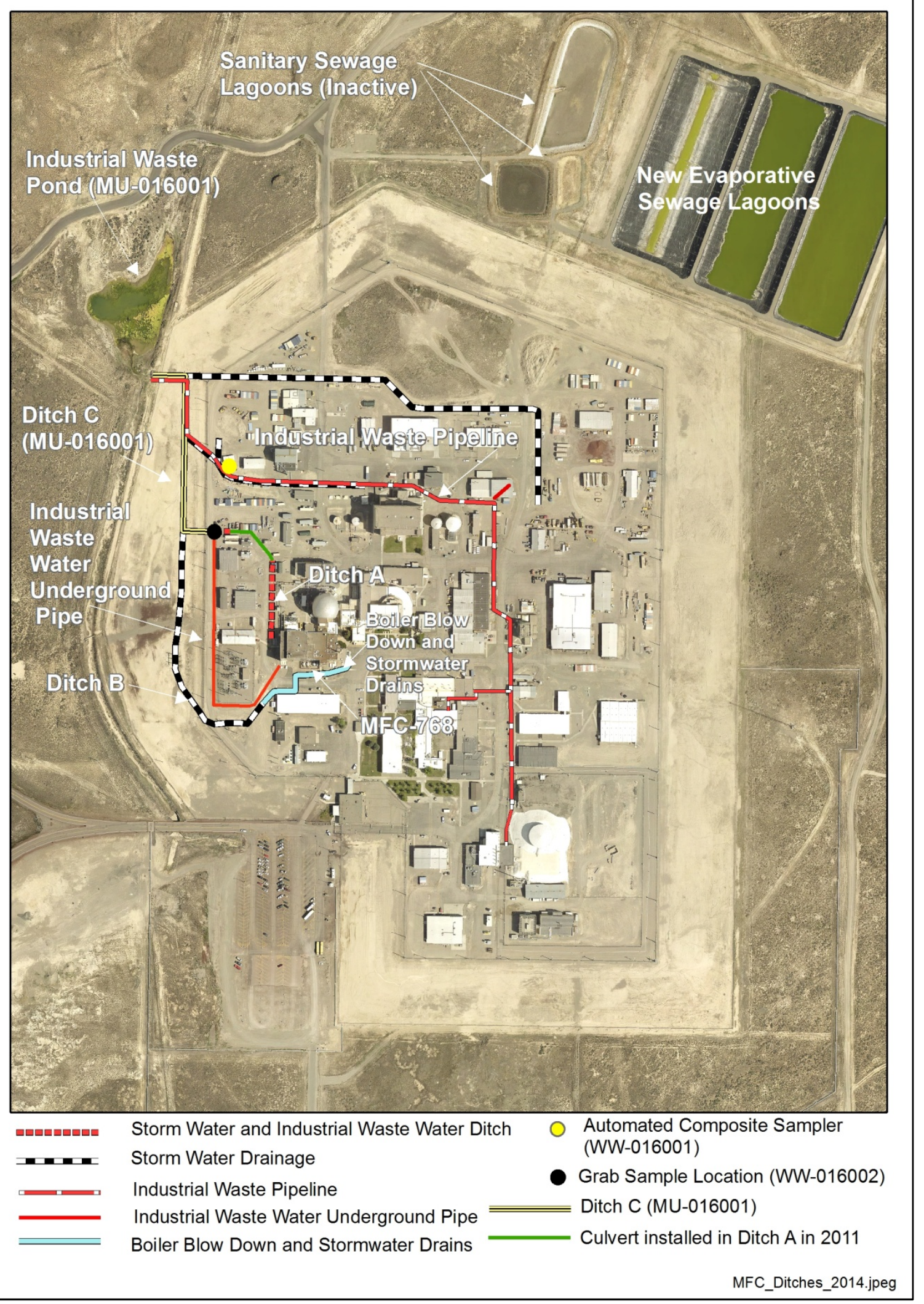

Figure 1. MFC Industrial Waste Ditch and Industrial Waste Pond. 


\section{EFFLUENT MONITORING}

This section describes the sampling and analytical methods used in the MFC IWP and Ditch C monitoring programs. Effluent monitoring and flow data is provided.

\subsection{Sampling Program and Analytical Methods}

Environmental professionals from Regulatory and Monitoring Services (R\&MS) perform the monthly and quarterly effluent monitoring required in Section $\mathrm{G}$ of the permit. Effluent samples were collected monthly from the Industrial Waste Pipeline (sampling location WW-016001) prior to discharge to the IWP (Figure 1). In addition, quarterly grab samples were collected from the effluent discharging into Ditch C from the Industrial Waste Water Underground Pipe (WW-016002). All samples were collected according to established programmatic sampling procedures.

Effluent samples are typically collected during a preselected week following a randomly generated sampling schedule to represent normal operating conditions. On occasion, the sampling schedule must be changed. This was the case for the sampling events originally scheduled for the Industrial Waste Pipeline for February $4^{\text {th }}$ and $5^{\text {th }}$ and March $4^{\text {th }}$ and $5^{\text {th }}$ and for Ditch C scheduled for January $27^{\text {th }}$.

On February $5^{\text {th }}$, not enough sample volume was collected from the Industrial Waste Pipeline due to a frozen sample line. Sample collection was rescheduled for February $10^{\text {th }}$ and $11^{\text {th }}$. Samples were collected on February $11^{\text {th }}$ (Table 1$)$.

Due to a planned power outage, samples could not be collected from the Industrial Waste Pipeline in March as scheduled. The sampling event was rescheduled for March $10^{\text {th }}$ and $11^{\text {th }}$. Samples were collected on March 11, 2014 (Table 1).

An attempt was made to collect the first quarter Ditch C sample in January. However, there was no wastewater flowing at the Ditch $\mathrm{C}$ collection point. This sampling event was rescheduled to February 12 , 2014 (Table 2).

All samples were analyzed using methods identified in 40 Code of Federal Regulations (CFR) 136, "Guidelines Establishing Test Procedures for the Analysis of Pollutants," 40 CFR 141, "National Primary Drinking Water Regulations," 40 CFR 143, "National Secondary Drinking Water Regulations," or approved by the DEQ.

The hydrogen ion activity $(\mathrm{pH})$ of the samples was measured with a calibrated meter at the time of sample collection. All other permit required samples were submitted under full chain of custody to Southwest Research Institute's Analytical and Environmental Chemistry Department located in San Antonio, Texas for analyses.

\subsection{Effluent Monitoring Results}

\subsubsection{Industrial Waste Pipeline}

Effluent samples were collected monthly from the Industrial Waste Pipeline (WW-016001) prior to discharge to the IWP. All effluent samples were collected as 24-hour flow proportional composite samples as required by the permit. Table 1 summarizes the analytical results for the monthly samples collected from the Industrial Waste Pipeline.

Section F of the IWRP specifies effluent permit limits based on a 30-day average for total nitrogen (TN) and total suspended solids (TSS) of $20 \mathrm{mg} / \mathrm{L}$ and $100 \mathrm{mg} / \mathrm{L}$, respectively. Total nitrogen is calculated as the sum of total Kjeldahl nitrogen and nitrate plus nitrite, as nitrogen. The maximum TN was $3.078 \mathrm{mg} / \mathrm{L}$ in the July 2014 sample. All TSS results were below the laboratory instrument detection limit of $4 \mathrm{mg} / \mathrm{L}$ with the exception of the August 2014 sample concentration of $7.7 \mathrm{mg} / \mathrm{L}$. No permit limits are specified for the other required analytes at the Industrial Waste Pipeline. 
In the 2013 annual report, it was identified that several metals (e.g., iron) showed elevated levels in June and October 2013 samples. Figure 2 shows the iron, lead, and manganese levels in samples collected from the Industrial Waste Pipeline for the period of November 2010 through October 2014.

For comparison purposes only, Figure 2 shows the Ground Water Quality Rule (IDAPA 58.01.11) Primary Constituent Standard (PCS) for lead and the Secondary Constituent Standards (SCSs) for iron and manganese.

Since November 2010, iron concentrations in the Industrial Waste Pipeline samples have only been above the SCS for iron three times (Figure 2). These occurred in June and October 2013 samples and the August 2014 sample. Typical iron concentrations in the Industrial Waste Pipeline are approximately 1/3 of the SCS for iron.

Lead concentrations from samples collected from the Industrial Waste Pipeline have only been above the PCS one time since November 2010. This occurred in the October 2013 sample. As shown in Figure 2 , iron, lead, and manganese all had elevated levels in the October 2013 sample.

Manganese (Figure 2) concentrations in the Industrial Waste Pipeline have been consistently below the SCS. Manganese concentrations in the samples were consistently at or near the laboratory instruments minimum detection limit, although occasional spikes did occur.

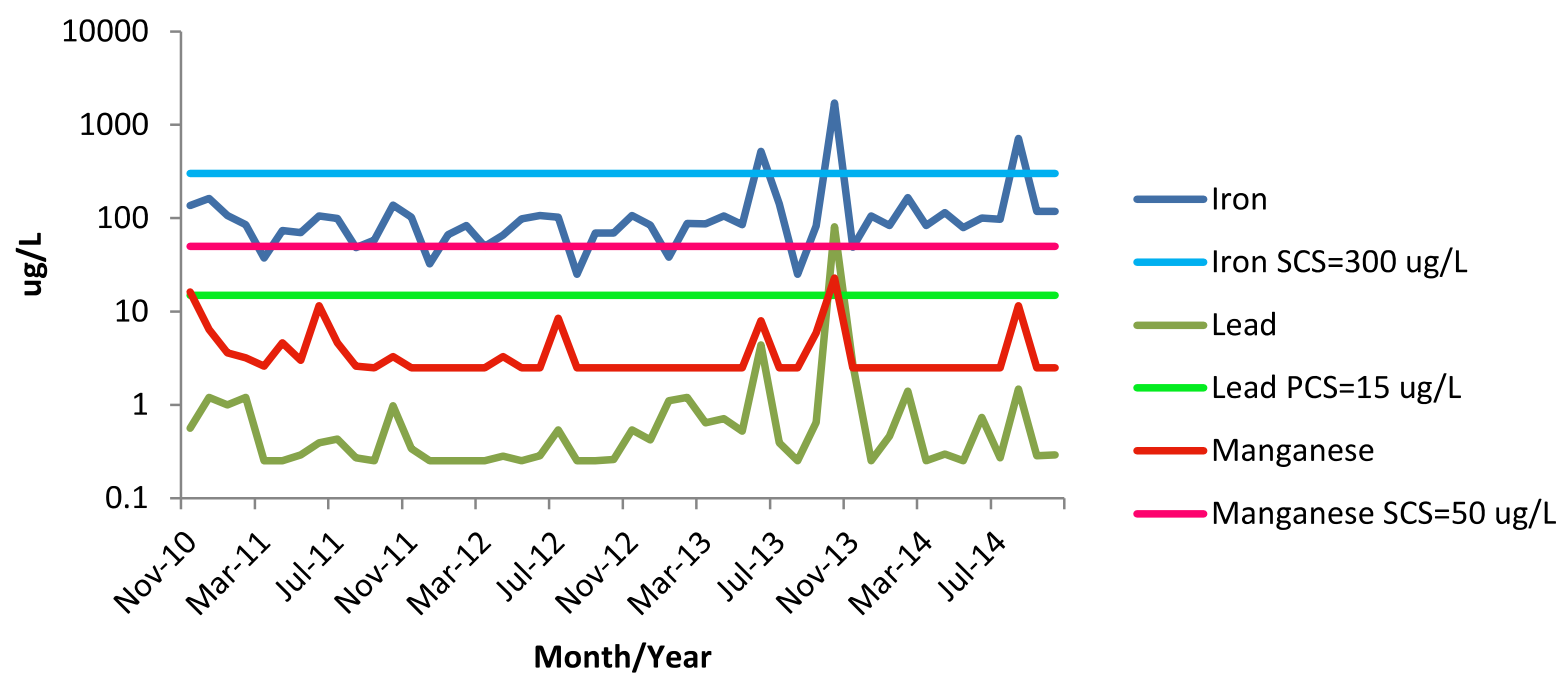

Figure 2. Iron, lead, and manganese concentrations in the Industrial Waste Pipeline from November 2010 through October 2014.

NOTE: For sample results below the laboratory instrument detection levels shown in Figure 2, the detection level was used. During months where a duplicate sample was collected, the regular sample and duplicate sample results were averaged.

\subsubsection{Effluent to Ditch C}

Grab samples were collected quarterly from the wastewater discharging into Ditch $\mathrm{C}$ from the Industrial Waste Water Underground Pipe (Figure 1). The analytical results are summarized in Table 2. 
Section F of the IWRP specifies effluent permit limits based on a 30-day average for total nitrogen (TN) and total suspended solids (TSS) of $20 \mathrm{mg} / \mathrm{L}$ and $100 \mathrm{mg} / \mathrm{L}$, respectively. Total nitrogen is calculated as the sum of total Kjeldahl nitrogen and nitrate plus nitrite nitrogen. The maximum TN was $8.98 \mathrm{mg} / \mathrm{L}$ in the third quarter (August 2014) sample. The maximum TSS result of $182 \mathrm{mg} / \mathrm{L}$, from the sample collected in the second quarter, exceeded the IWRP limit. There are no effluent permit limits specified for the other required analytes.

On July 28, 2014, a follow up sample to the second quarter (June 11, 2014) TSS sample was collected. The TSS concentration in the July sample was $6 \mathrm{mg} / \mathrm{L}$. TSS concentrations for the other three quarters were below the laboratory instrument minimum detection level of $4 \mathrm{mg} / \mathrm{L}$. See Section 5.2 for additional TSS information. 


\begin{tabular}{|c|c|c|c|c|c|c|c|c|c|c|c|c|c|c|c|c|c|c|}
\hline $\begin{array}{l}5 \\
\overline{0} \\
\overline{0} \\
0\end{array}$ & 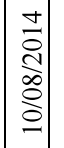 & ָे & \begin{tabular}{l}
$\stackrel{\circ}{0}$ \\
\hdashline
\end{tabular} & 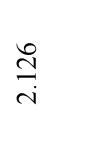 & $\underset{\forall}{P}$ & $\tilde{n}$ & $\stackrel{\partial}{\partial}$ & $\begin{array}{l}\text { on } \\
\overbrace{0}^{2}\end{array}$ & $\stackrel{2}{\therefore}$ & 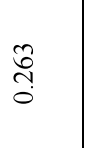 & $\begin{array}{l}\stackrel{\infty}{ } \\
\stackrel{0}{ }\end{array}$ & $\begin{array}{l}\text { D } \\
\text { in }\end{array}$ & 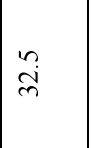 & $\stackrel{?}{\circ}$ & $\vec{i}$ & $\stackrel{\infty}{=}$ & ते & $\begin{array}{l}? \\
n \\
i\end{array}$ \\
\hline 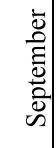 & 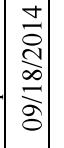 & $\underset{\sim}{\sim}$ & $\stackrel{n}{n}$ & $\underset{i}{\stackrel{g}{i}}$ & $\underset{\forall}{\supset}$ & $\hat{m}$ & $\tilde{b}$ & $\begin{array}{l}n \\
\hat{n} \\
0\end{array}$ & $\stackrel{ }{i}$ & $\begin{array}{l}\tilde{N} \\
0 \\
0 \\
0\end{array}$ & $\stackrel{\infty}{\infty}$ & $\begin{array}{l}\text { P } \\
0 \\
\text { in }\end{array}$ & 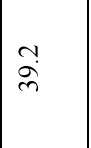 & 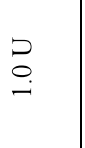 & ले & $\stackrel{\infty}{=}$ & 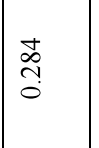 & $\begin{array}{l}\stackrel{p}{n} \\
\text { in }\end{array}$ \\
\hline $\begin{array}{l}\vec{y} \\
\vec{z} \\
\vec{z} \\
\vec{z}\end{array}$ & 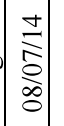 & 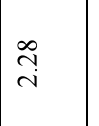 & ふָ & $\underset{\substack{n \\
i}}{\stackrel{N}{n}}$ & $\stackrel{\therefore}{\therefore}$ & ర్ల & $\stackrel{i}{i}$ & $\begin{array}{l}\vec{J} \\
\stackrel{0}{0}\end{array}$ & $\frac{2}{\infty}$ & $\bar{m}$ & $\stackrel{\infty}{\infty}$ & $\begin{array}{l}\text { P } \\
\text { in }\end{array}$ & $\mid \begin{array}{l}\infty \\
\stackrel{\sim}{\sigma}\end{array}$ & $\stackrel{?}{\stackrel{P}{-}}$ & $\stackrel{\infty}{=}$ & $\stackrel{m}{\sim}$ & 守 & $\stackrel{\circ}{\stackrel{\Xi}{=}}$ \\
\hline 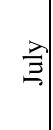 & $\mid \frac{9}{2}$ & $\stackrel{\infty}{i}$ & 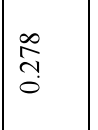 & 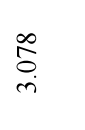 & $\stackrel{\succ}{\succ}$ & $\underset{\sim}{~}$ & $\hat{8}$ & $\stackrel{\infty}{\stackrel{\infty}{0}}$ & $\mid \begin{array}{c}\infty \\
\infty \\
\sim\end{array}$ & 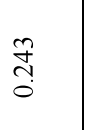 & $\begin{array}{l}\mathbf{\sim} \\
\text { ते }\end{array}$ & $\begin{array}{l}0 \\
0 \\
\text { in }\end{array}$ & $\mid \begin{array}{l}\infty \\
0 \\
0\end{array}$ & $\stackrel{?}{?}$ & $\begin{array}{l}0 \\
n \\
i \\
i\end{array}$ & $\stackrel{n}{\stackrel{n}{\alpha}}$ & 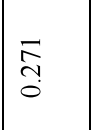 & $\begin{array}{l}? \\
\text { ñ } \\
i\end{array}$ \\
\hline$\stackrel{\Xi}{\Xi}$ & 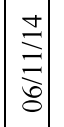 & $\stackrel{i}{i}$ & $\frac{2}{0}$ & $\stackrel{\vec{\sim}}{\stackrel{\sim}{v}}$ & $\stackrel{P}{q}$ & $\stackrel{\infty}{\sim}$ & $\begin{array}{l}\infty \\
\underset{\sim}{\text { in }}\end{array}$ & $\frac{2}{2}$ & à & $\underset{\Xi}{\tilde{\sigma}}$ & $\bar{\infty}$ & $\begin{array}{l}0 \\
0 \\
\text { in }\end{array}$ & $\hat{b}$ & $\stackrel{?}{?} \underset{-}{0}$ & $\begin{array}{l}2 \\
n \\
i \\
i\end{array}$ & 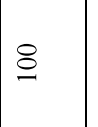 & 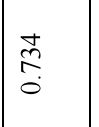 & $\begin{array}{l}D \\
i n \\
i\end{array}$ \\
\hline$\stackrel{\vec{m}}{\mathrm{~m}}$ & $\mid$\begin{tabular}{l|}
$\stackrel{+}{\infty}$ \\
$\stackrel{0}{0}$ \\
$\forall$ \\
$\delta$
\end{tabular} & 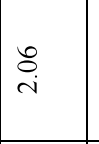 & $\frac{2}{0}$ & $\begin{array}{l}\stackrel{\circ}{\vec{v}} \\
\vec{v}\end{array}$ & $\stackrel{?}{\succ}$ & $\bar{m}$ & $\frac{n}{6}$ & $\stackrel{N}{0}$ & ㄱ. & \begin{tabular}{l}
0 \\
के \\
\multirow{0}{0}{} \\
0
\end{tabular} & $\begin{array}{l}\underset{\infty}{\infty} \\
\infty\end{array}$ & $\begin{array}{l}\text { D } \\
0 \\
\text { in }\end{array}$ & $\hat{\sigma}$ & $\stackrel{?}{?}$ & $\begin{array}{l}0 \\
n \\
i\end{array}$ & $\vec{a}$ & $\begin{array}{l}2 \\
2 \\
\\
0\end{array}$ & $\begin{array}{l}p \\
\text { n } \\
i\end{array}$ \\
\hline$\overline{\mathrm{c}}$ & 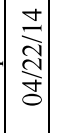 & $\stackrel{d}{d}$ & $\frac{2}{0}$ & $\underset{\tilde{N}}{\stackrel{v}{v}}$ & $\underset{\forall}{P}$ & $\underset{\sim}{\stackrel{\sim}{~}}$ & $\stackrel{\Delta}{\vec{N}}$ & $\begin{array}{l}8 \\
0 \\
0 \\
0\end{array}$ & $\stackrel{2}{r}$ & $\begin{array}{l}\text { Oo } \\
\stackrel{0}{0} \\
0 \\
0\end{array}$ & $\stackrel{+}{2}$ & $\begin{array}{l}\vec{D} \\
\text { in } \\
\text { in }\end{array}$ & $\stackrel{m}{\text { m }}$ & 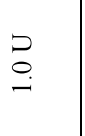 & $\begin{array}{l}P \\
\stackrel{n}{n} \\
i\end{array}$ & $\cong$ & ڤั) & $\stackrel{?}{n}$ \\
\hline $\begin{array}{l}\frac{-}{0} \\
\frac{0}{\pi} \\
\Sigma\end{array}$ & 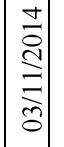 & $\vec{i}$ & $\frac{D}{0}$ & $\tilde{\tilde{V}}$ & $\stackrel{\nabla}{\supset}$ & $\stackrel{\sim}{\approx}$ & $\stackrel{n}{2}$ & $\begin{array}{l}8 \\
0 \\
0 \\
0\end{array}$ & $\stackrel{?}{r}$ & $\stackrel{\infty}{=}$ & 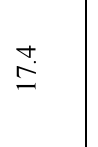 & $\begin{array}{l}\vec{D} \\
\text { in } \\
\text { in }\end{array}$ & $\underset{\sim}{\tilde{m}}$ & 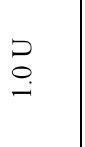 & $\begin{array}{l}D \\
n \\
i \\
i\end{array}$ & $\bar{\infty}$ & $\begin{array}{l}0 \\
\hat{n} \\
0 \\
0\end{array}$ & $\begin{array}{l}p \\
\text { n } \\
i\end{array}$ \\
\hline 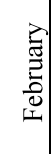 & 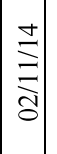 & $\begin{array}{cc}\simeq & a \\
& a \\
c\end{array}$ & $\left|\begin{array}{ll}2 & 9 \\
0 & 0 \\
0 & 0\end{array}\right|$ & $\begin{array}{ll}\tilde{N} & \sigma \\
\vec{v} & \vec{v}\end{array}$ & 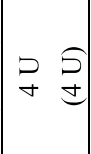 & 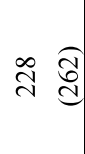 & 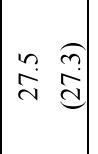 & $\begin{array}{ll}\infty & \widehat{0} \\
0 & 0 \\
0 & \stackrel{\theta}{*}\end{array}$ & $\stackrel{n}{\sim}$ & 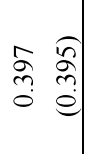 & 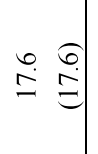 & $\left.\mid \begin{array}{cc}2 & 5 \\
0 & 0 \\
i & 0\end{array}\right]$ & {$\left[\begin{array}{cc}n & 0 \\
2 & \infty \\
\text { m) } & 0\end{array}\right.$} & $\begin{array}{cc}D & 0 \\
\circ & 0 \\
- & 0\end{array}$ & $\left|\begin{array}{ll}D & 0 \\
n & n \\
i & d\end{array}\right|$ & $\stackrel{\stackrel{a}{+}}{=}$ & $=0$ & $\begin{array}{ll}D & S \\
n & n \\
i & c\end{array}$ \\
\hline 跑 & $\mid$\begin{tabular}{|}
$\frac{7}{6}$ \\
$\stackrel{0}{0}$ \\
\end{tabular} & $\frac{n}{i}$ & $\frac{2}{0}$ & $\stackrel{\sim}{\tilde{V}}$ & $\stackrel{\nabla}{\supset}$ & $\underset{\sim}{\stackrel{D}{i}}$ & के & $\begin{array}{l}\infty \\
\infty \\
0 \\
0 \\
0\end{array}$ & $\stackrel{8}{\sim}$ & $\begin{array}{l}\tilde{\delta} \\
\delta \\
0\end{array}$ & $\stackrel{m}{2}$ & in & $\underset{m}{\ddot{n}}$ & $\stackrel{?}{\circ}$ & $\begin{array}{l}D \\
i n \\
i\end{array}$ & $\stackrel{\dot{\infty}}{\dot{\infty}}$ & $\begin{array}{l}\text { 广). } \\
0\end{array}$ & $\begin{array}{l}? \\
\text { n } \\
i\end{array}$ \\
\hline 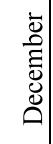 & 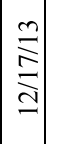 & $\underset{\text { Cे }}{i}$ & $\begin{array}{l}\frac{1}{6} \\
0 \\
0\end{array}$ & $\underset{⿱ 亠}{\stackrel{ \pm}{S}}$ & $\underset{\forall}{\supset}$ & $\vec{n}$ & $\stackrel{m}{\tilde{n}}$ & \begin{tabular}{l}
\multirow{0}{0}{} \\
0 \\
0
\end{tabular} & $\stackrel{m}{r}$ & $\begin{array}{l}\stackrel{0}{7} \\
0\end{array}$ & $\stackrel{\circ}{\stackrel{\circ}{\Sigma}}$ & in & $\frac{\infty}{m}$ & 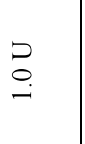 & $\begin{array}{l}? \\
\stackrel{2}{n} \\
i\end{array}$ & $\stackrel{\imath}{\varrho}$ & $\begin{array}{l}\overrightarrow{2} \\
\text { ñ } \\
0\end{array}$ & $\begin{array}{l}? \\
\text { n } \\
i\end{array}$ \\
\hline $\begin{array}{l}\bar{\Xi} \\
\bar{\Xi} \\
\overline{0} \\
z \\
z\end{array}$ & 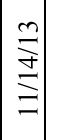 & $\stackrel{n}{a}$ & $\begin{array}{l}3 \\
\frac{3}{0}\end{array}$ & $\stackrel{n}{\stackrel{\sim}{v}}$ & $\stackrel{\nabla}{\supset}$ & 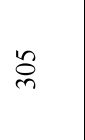 & $\bar{n}$ & $\begin{array}{l}\hat{n} \\
i \\
0\end{array}$ & ָे & $\frac{\infty}{0}$ & $\stackrel{\simeq}{I}$ & in & $\begin{array}{l}0 \\
\stackrel{m}{m}\end{array}$ & 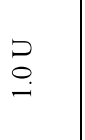 & $\begin{array}{l}D \\
\sim \\
i\end{array}$ & $\begin{array}{l}\infty \\
\stackrel{\infty}{\sigma}\end{array}$ & $\hat{i}$ & $\begin{array}{l}? \\
n \\
i\end{array}$ \\
\hline $\begin{array}{l}\bar{n} \\
\sum_{0} \\
0 \\
\overline{0} \\
\bar{n} \\
\tilde{n}\end{array}$ & 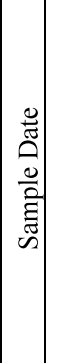 & 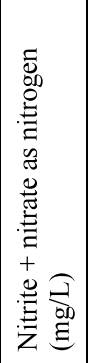 & 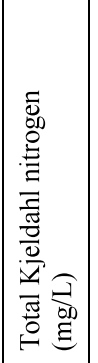 & 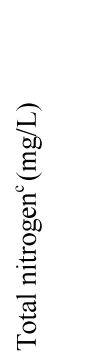 & 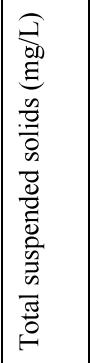 & 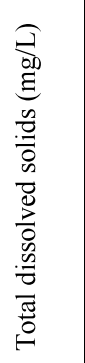 & 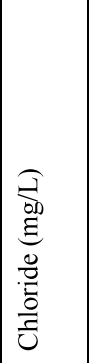 & 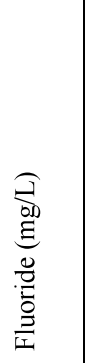 & & 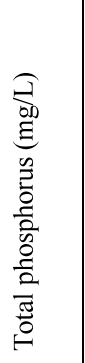 & 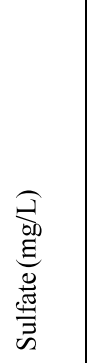 & 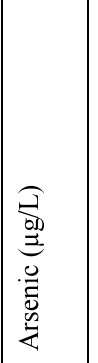 & 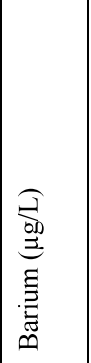 & 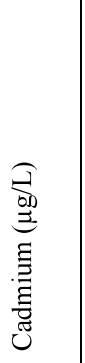 & 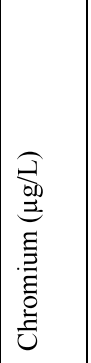 & 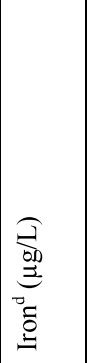 & 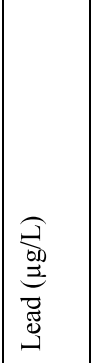 & 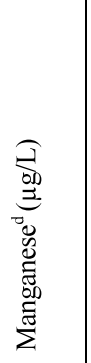 \\
\hline
\end{tabular}




\begin{tabular}{|c|c|c|c|c|c|c|c|}
\hline 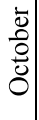 & $\begin{array}{l}\stackrel{\Xi}{\infty} \\
\stackrel{0}{0}\end{array}$ & $\begin{array}{l}\text { Dे } \\
\text { กิ } \\
0\end{array}$ & $\begin{array}{l}\overrightarrow{\widehat{C}} \\
0 \\
0\end{array}$ & $\begin{array}{l}\text { P } \\
\text { in }\end{array}$ & $\begin{array}{l}8 \\
\frac{8}{\sim} \\
-1\end{array}$ & $\begin{array}{l}\stackrel{n}{m} \\
\stackrel{n}{n}\end{array}$ & 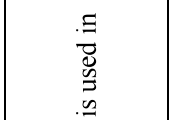 \\
\hline 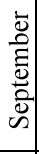 & 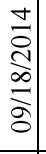 & $\begin{array}{l}\text { D } \\
\text { กิ } \\
0\end{array}$ & $\begin{array}{l}\text { ¿े } \\
\text { 。 }\end{array}$ & $\begin{array}{l}? \\
\text { in } \\
\text { in }\end{array}$ & 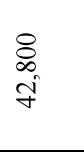 & $\stackrel{+}{ \pm}$ & 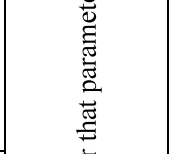 \\
\hline 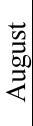 & 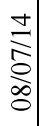 & $\begin{array}{l}\text { D } \\
\text { กุ } \\
0\end{array}$ & $\begin{array}{l}\stackrel{J}{0} \\
\stackrel{0}{0}\end{array}$ & $\begin{array}{l}\text { P } \\
\text { in }\end{array}$ & 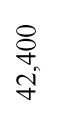 & $\approx$ & 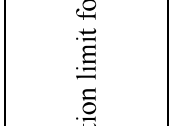 \\
\hline 글 & $\underset{⿱ 亠 䒑}{\frac{5}{5}}$ & $\begin{array}{l}\text { D } \\
\text { กิ } \\
0\end{array}$ & $\underset{0}{\stackrel{H}{0}}$ & $\begin{array}{l}\text { D } \\
\text { in } \\
\text { in }\end{array}$ & $\begin{array}{l}8 \\
\text { qे } \\
\text { ót }\end{array}$ & $\begin{array}{l}\infty \\
\infty \\
\infty \\
\infty\end{array}$ & 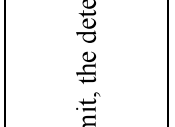 \\
\hline$\stackrel{\Xi}{\Xi}$ & 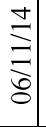 & 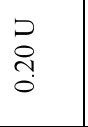 & $\begin{array}{l}? \\
n \\
o\end{array}$ & $\begin{array}{l}\text { P } \\
\text { in }\end{array}$ & 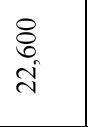 & $\stackrel{t}{\ominus}$ & 㺼 \\
\hline 鹿 & $\begin{array}{c}\frac{\pi}{a} \\
\substack{0 \\
\vdots}\end{array}$ & $\begin{array}{l}\text { ? } \\
\text { त̂ } \\
0\end{array}$ & $\begin{array}{l}P \\
n \\
o\end{array}$ & $\begin{array}{l}\text { P } \\
\text { in }\end{array}$ & $\frac{8}{\frac{8}{8}}$ & $\simeq$ & 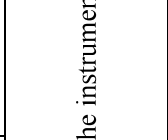 \\
\hline$\overline{\mathrm{a}}$ & 辛 & 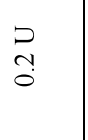 & $\begin{array}{l}P \\
n \\
0\end{array}$ & $\begin{array}{l}P \\
\text { in } \\
\text { in }\end{array}$ & $\begin{array}{l}8 \\
\infty \\
\infty \\
\infty \\
\infty\end{array}$ & $\simeq$ & 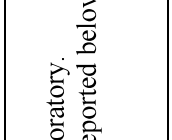 \\
\hline 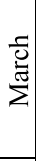 & 离 & ? & $\bar{n}$ & $\begin{array}{l}\text { P. } \\
\text { in }\end{array}$ & $\begin{array}{l}8 \\
: \\
2\end{array}$ & $\stackrel{\circ}{=}$ & 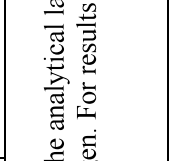 \\
\hline 悹 & 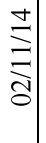 & $\left|\begin{array}{ll}0 & 0 \\
0 & 0 \\
i & 0 \\
0 & 0\end{array}\right|$ & 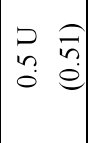 & $\left|\begin{array}{cc}0 & 0 \\
0 & 0 \\
\text { in } & \dot{n}\end{array}\right|$ & $\left|\begin{array}{cc}8 & 8 \\
\hdashline & d \\
\dot{d} & d\end{array}\right|$ & 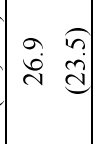 & 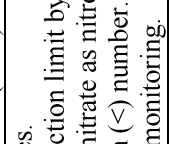 \\
\hline 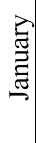 & 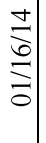 & ? & $\stackrel{0}{\dot{m}}$ & $\begin{array}{l}\vec{P} \\
\text { in } \\
\text { in }\end{array}$ & $\begin{array}{l}8 \\
\text { r. } \\
\hat{n} \\
\text { in }\end{array}$ & $\stackrel{\stackrel{+}{\infty}}{\sim}$ & 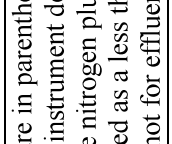 \\
\hline 范 & 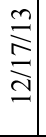 & D̊ & 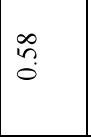 & $\begin{array}{l}\vec{P} \\
\text { in }\end{array}$ & $\begin{array}{l}8 \\
\infty \\
\infty \\
\text { in }\end{array}$ & $\infty$ & 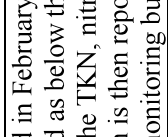 \\
\hline 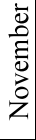 & $\stackrel{\substack{f \\
\Xi}}{=}$ & ? & : & $\begin{array}{l}\text { P } \\
\text { in }\end{array}$ & $\frac{8}{m^{n}}$ & $\underset{\infty}{+}$ & 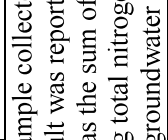 \\
\hline & 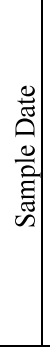 & 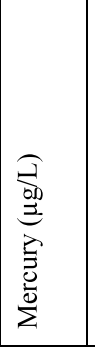 & 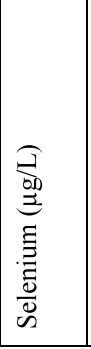 & 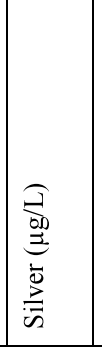 & 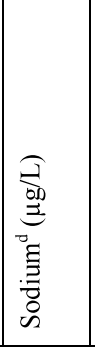 & 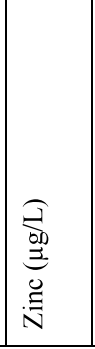 & 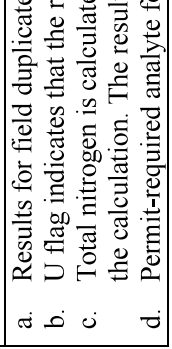 \\
\hline
\end{tabular}




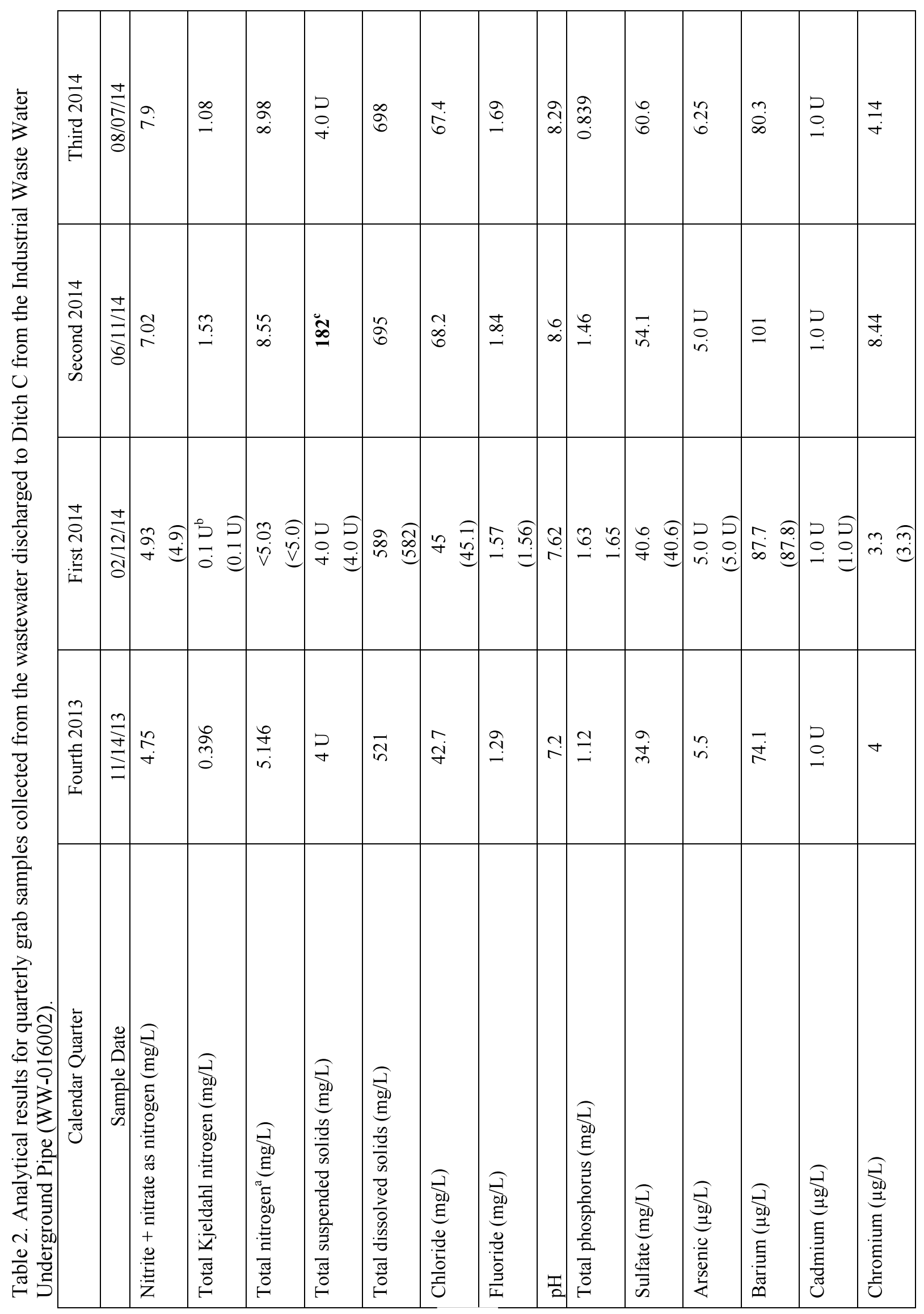




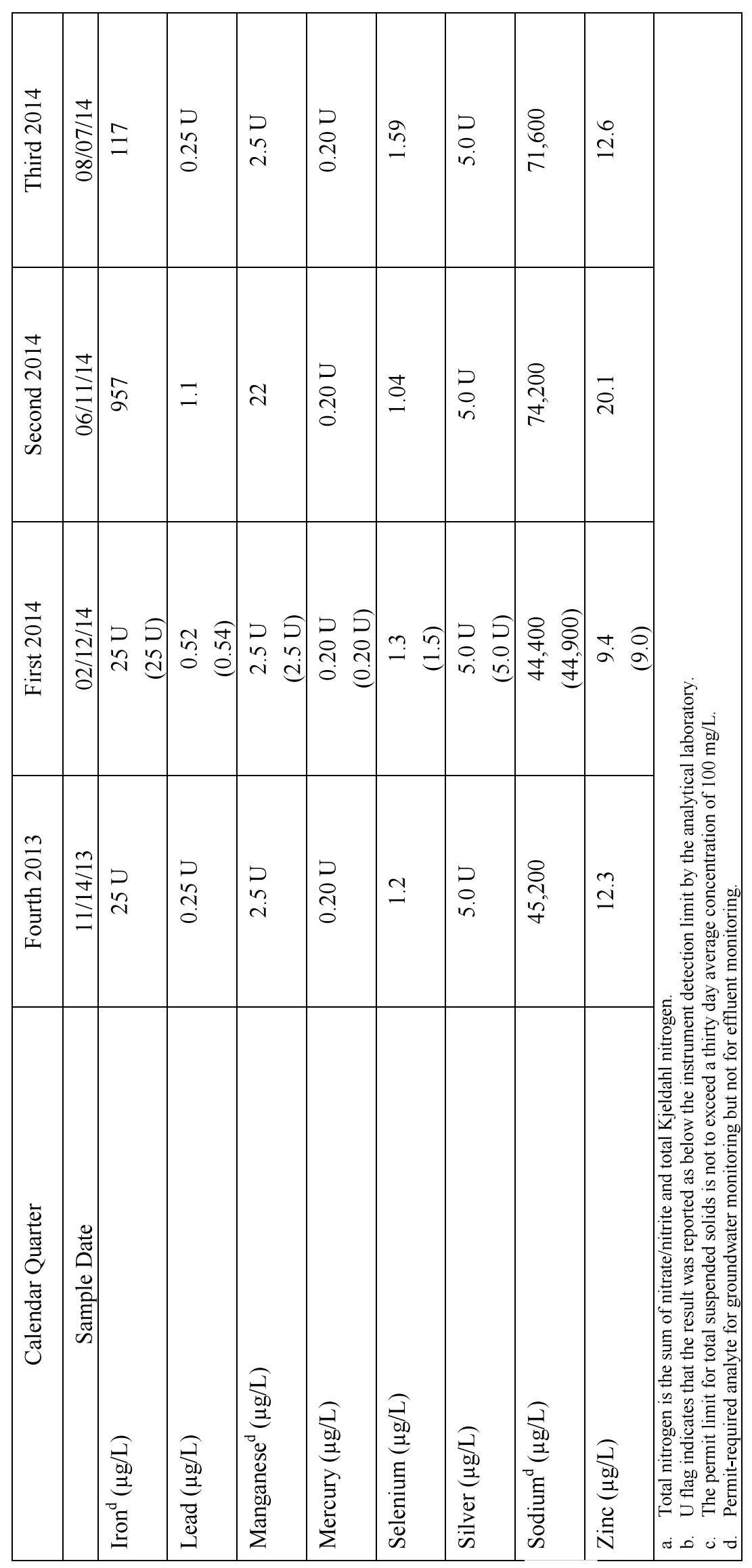




\subsection{Flow Volumes and Hydraulic Loading Rates}

\subsubsection{Industrial Waste Pipeline to Industrial Waste Pond}

Section $\mathrm{G}$ of the permit requires a flow meter to measure the monthly volume of flow to the Industrial Waste Pond from the Industrial Waste Pipeline to the nearest 0.000 million gallons. Monthly flow volumes are recorded from the flow meter located at the effluent sampling point WW-016001. Table 3 summarizes the monthly and annual flow data.

Table 3. Volume of wastewater discharged from the Industrial Waste Pipeline to the Industrial Waste Pond.

\begin{tabular}{|c|c|c|}
\hline & $\begin{array}{l}\text { Average } \\
\left(\mathrm{gpm}^{\mathrm{a}}\right)\end{array}$ & $\begin{array}{l}\text { Total } \\
\left(\mathrm{MG}^{\mathrm{b}}\right)\end{array}$ \\
\hline November 2013 & 29.0 & 1.254 \\
\hline December 2013 & 15.7 & 0.699 \\
\hline January 2014 & 17.4 & 0.776 \\
\hline February 2014 & 17.8 & 0.718 \\
\hline March 2014 & 17.2 & 0.767 \\
\hline April 2014 & 24.7 & 1.068 \\
\hline May 2014 & 17.7 & 0.790 \\
\hline June 2014 & 16.6 & 0.717 \\
\hline July 2014 & 16.4 & 0.733 \\
\hline August 2014 & 16.2 & 0.722 \\
\hline September 2014 & 16.7 & 0.722 \\
\hline October 2014 & 13.7 & 0.613 \\
\hline $\begin{array}{l}\text { TOTAL } \\
\text { a. gpm-gallons per minute. } \\
\text { b. MG_-million gallons. }\end{array}$ & & 9.580 \\
\hline
\end{tabular}

\subsubsection{Industrial Waste Water Underground Pipeline to Ditch C}

As required by Section $G$ of the permit, the monthly flow from the Industrial Waste Water Underground Pipeline to Ditch C was visually estimated by Environmental, Safety, and Health personnel. Table 4 summarizes the monthly and annual flow data. 
Table 4. Volume of wastewater discharged from the Industrial Waste Water Underground Pipe to Ditch C.

\begin{tabular}{lcc}
\hline & $\begin{array}{c}\text { Average }^{\mathrm{a}} \\
\left(\mathrm{gpm}^{\mathrm{b}}\right)\end{array}$ & $\begin{array}{c}\text { Total } \\
\left(\mathrm{MG}^{\mathrm{c}}\right)\end{array}$ \\
\hline November 2013 & 1 & 0.043 \\
December 2013 & 1 & 0.045 \\
January 2014 & 1 & 0.045 \\
February 2014 & 1 & 0.040 \\
March 2014 & 1 & 0.045 \\
April 2014 & 1 & 0.043 \\
May 2014 & 1 & 0.045 \\
June 2014 & 1 & 0.043 \\
July 2014 & 1 & 0.045 \\
August 2014 & 1 & 0.045 \\
September 2014 & 1 & 0.043 \\
October 2014 & 1 & 0.045 \\
TOTAL & & 0.527 \\
a. Average of multiple visual flow estimates during the month. & \\
b. gpm-gallons per minute. & & \\
c. MG-million gallons. & & \\
\hline
\end{tabular}

\subsubsection{Summary}

The permit (Section F) specifies the following:

- Application season is year round.

- Maximum hydraulic loading rate is 17 million gallons (MG) per year.

Total effluent flow volume was an estimated 10.11 MG for the reporting period. 


\section{GROUNDWATER MONITORING}

The groundwater monitoring sections provide information concerning the INL sampling program, analytical methods used, monitoring results, and water table information.

\subsection{Sampling Program}

The IWRP for the MFC Industrial Waste Ditch (Ditch C) and Pond identifies three INL compliance wells. The permit requires the collection of groundwater samples in April or May and September or October.

In 2014, R\&MS personnel collected groundwater samples in April, May, and September. As scheduled, samples were collected from wells ANL-MON-A-013 and ANL-MON-A-014 on April 22, 2014 and April 21, 2014, respectively. However, R\&MS personnel were unable to collect samples from well ANL-MON-A-012 in April 2014 because the pump was inoperable. The pump was replaced with a new "like for like" pump and samples were collected from well ANL-MON-A-012 on May 27, 2014.

The R\&MS personnel use project-specific sampling and analysis plans and procedures that govern sampling activities and quality control protocols. The permit identifies a specified list of parameters that are to be analyzed in the groundwater samples. Constituent concentrations in the compliance wells are limited by primary constituent standards (PCS) and secondary constituent standards (SCS) specified in IDAPA 58.01.11, "Ground Water Quality Rule." All permit-required samples were collected as unfiltered samples with the exception of iron and manganese in wells that exceed the SCS for these parameters.

NOTE: Filtered samples for iron and manganese analysis are only required by the permit in the event the unfiltered sample results for these two parameters exceed the applicable SCSs.

The conductivity and $\mathrm{pH}$ of the samples were measured at the time of sample collection by R\&MS personnel using a calibrated meter. Groundwater temperature was also measured at the time of sample collection. All other permit required groundwater samples were submitted under full chain of custody to Southwest Research Institute's Analytical and Environmental Chemistry Department located in San Antonio, Texas for analyses.

\subsection{Analytical Methods}

Analytical methods specified in 40 CFR 141, "National Primary Drinking Water Regulations," 40 CFR 143, "National Secondary Drinking Water Regulations," 40 CFR 136, "Guidelines Establishing Test Procedures for the Analysis of Pollutants," or those approved by DEQ were used for analysis of all permit-required parameters.

\subsection{Monitoring Wells}

To evaluate potential impacts to groundwater from the wastewater discharges to the MFC Industrial Waste Pond, the permit requires that groundwater samples be collected from the Snake River Plain Aquifer at three monitoring wells (see Figures 4 and 5):

- $\quad$ ANL-MON-A-012 (GW-016001)

- $\quad$ ANL-MON-A-013 (GW-016002)

- $\quad$ ANL-MON-A-014 (GW-016003)

Prior to sampling, wells were purged a minimum of three casing volumes or one casing volume if three successive measurements for $\mathrm{pH}$ and specific conductance, taken at least one minute apart, had $\mathrm{pH}$ values within 0.2 units of each other and specific conductance readings within $10 \%$.

Updated well diagrams for all three wells were submitted to DEQ on September 23, 2014 (Miller 2014b). The diagrams show that new pumps were installed in wells ANL-MON-A-012 and ANL-MON- 
A-013 on May 27, 2014. Minor changes to well screen and casing lengths, updated brass cap elevations, etc., were also provided in the updated well diagrams.

\subsection{Groundwater Monitoring Results}

Table 5 shows the static water table elevations and depths to the water table, measured prior to purging and sampling, and the analytical results for all parameters specified by the permit for the three monitoring wells.

The concentrations of all permit-required analytes were below their respective groundwater quality standards in IDAPA 58.01.11 in the unfiltered samples collected during the 2014 reporting year (Table 5) with the exception of iron in all three wells.

Iron exceeded the SCS of $300 \mathrm{ug} / \mathrm{L}$ in wells ANL-MON-A-013 and ANL-MON-A-014 in the April unfiltered samples, in the May unfiltered sample from well ANL-MON-A-012, and in the September unfiltered sample from ANL-MON-A-13 (Figure 3). The highest unfiltered iron concentration was in the September sample from well ANL-MON-A-013 at 2,210 ug/L. All filtered iron results from the three wells were below the SCS.

Figure 3 shows the variability of the iron concentrations between the three wells and between the filtered and unfiltered samples from May 2010 through September 2014. The filtered sample results for iron in all three wells is at or near the laboratory instruments minimum detection level of $50 \mathrm{ug} / \mathrm{L}$.

To demonstrate compliance with the permit, if the unfiltered samples exceed the applicable SCS for iron and/or manganese, then the filtered sample results are used.

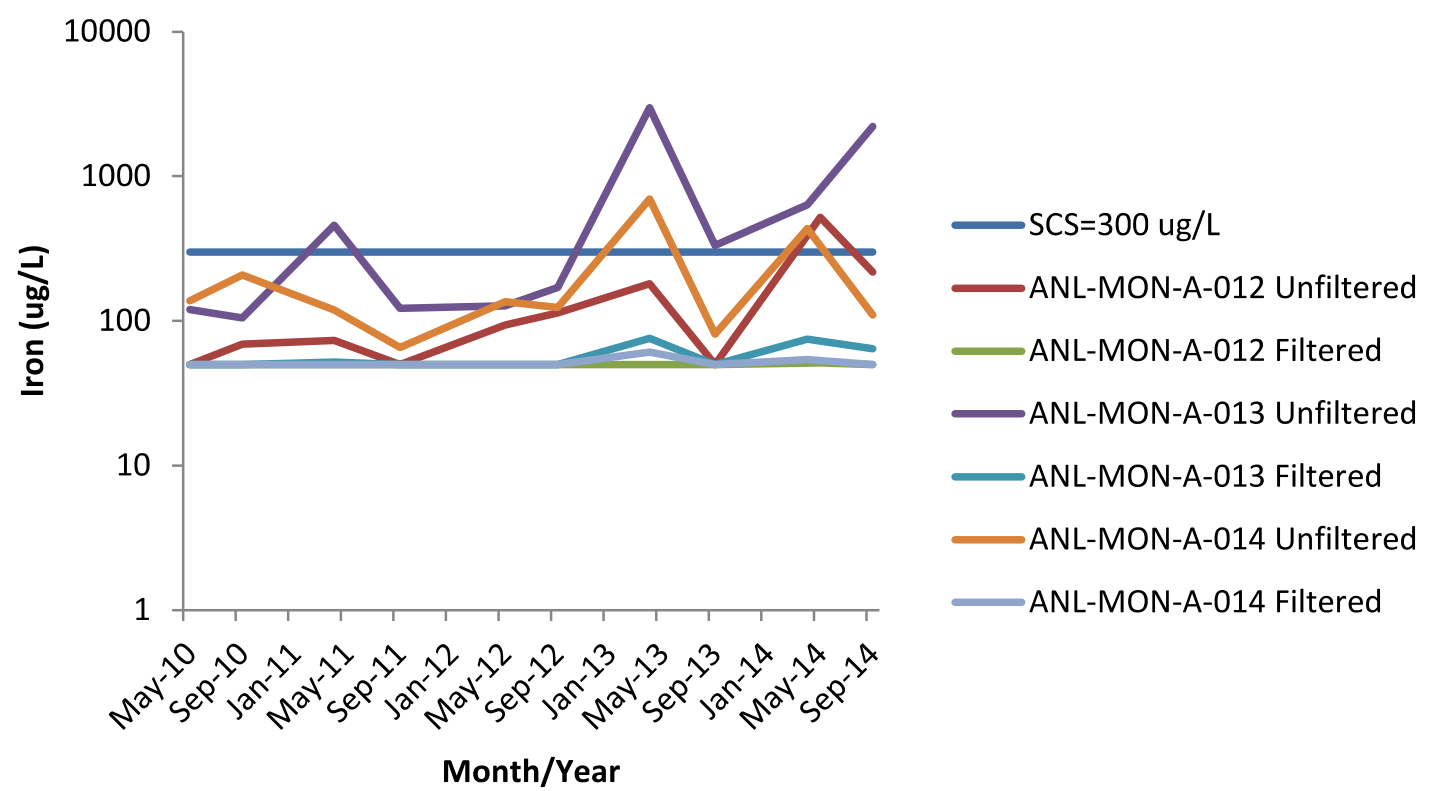

Figure 3. Iron concentrations in the unfiltered and filtered samples from the three aquifer monitoring wells for the period of May 2010 through September 2014.

NOTE: For iron sample results below the laboratory instrument detection levels shown in Figure 3, the detection level was used. During months where a duplicate sample was collected, the regular sample and duplicate sample results were averaged. 
As discussed in Section 4.3, new pumps were installed in wells ANL-MON-A-012 and ANL-MONA-013 in May 2014. After the old pumps were removed, the wells were videoed prior to installation of the new pumps. The videos show some corrosion of the 8 inch carbon steel casing, a reddish colored buildup on the stainless steel screen near the pump inlets, and sediment in the bottom of both wells that may contribute to the iron concentrations.

The iron concentrations in the effluent from the Industrial Waste Pipeline are typically less than half of the SCS for iron and below or slightly above the laboratory instrument detection level for manganese.

The concentrations of chloride, nitrate+nitrite (as nitrogen), phosphorus, and sodium appear to be elevated in the effluent from the Industrial Waste Pipeline and Ditch C; however, the concentrations of these constituents in the down gradient monitoring wells are nearly indistinguishable from the concentrations in the up gradient well (ANL-MON-A-012).

\subsection{Water Table Information}

Depth to water and water table elevations for the April, May, and September 2014, sampling events are shown in Figure 4 and Figure 5, respectively. The elevations are referenced to the National Geodetic Vertical Datum of 1929. In addition, the figures show the inferred general groundwater flow direction in the vicinity of the Materials and Fuels Complex. The general groundwater flow direction at the INL Site is to the southwest. 


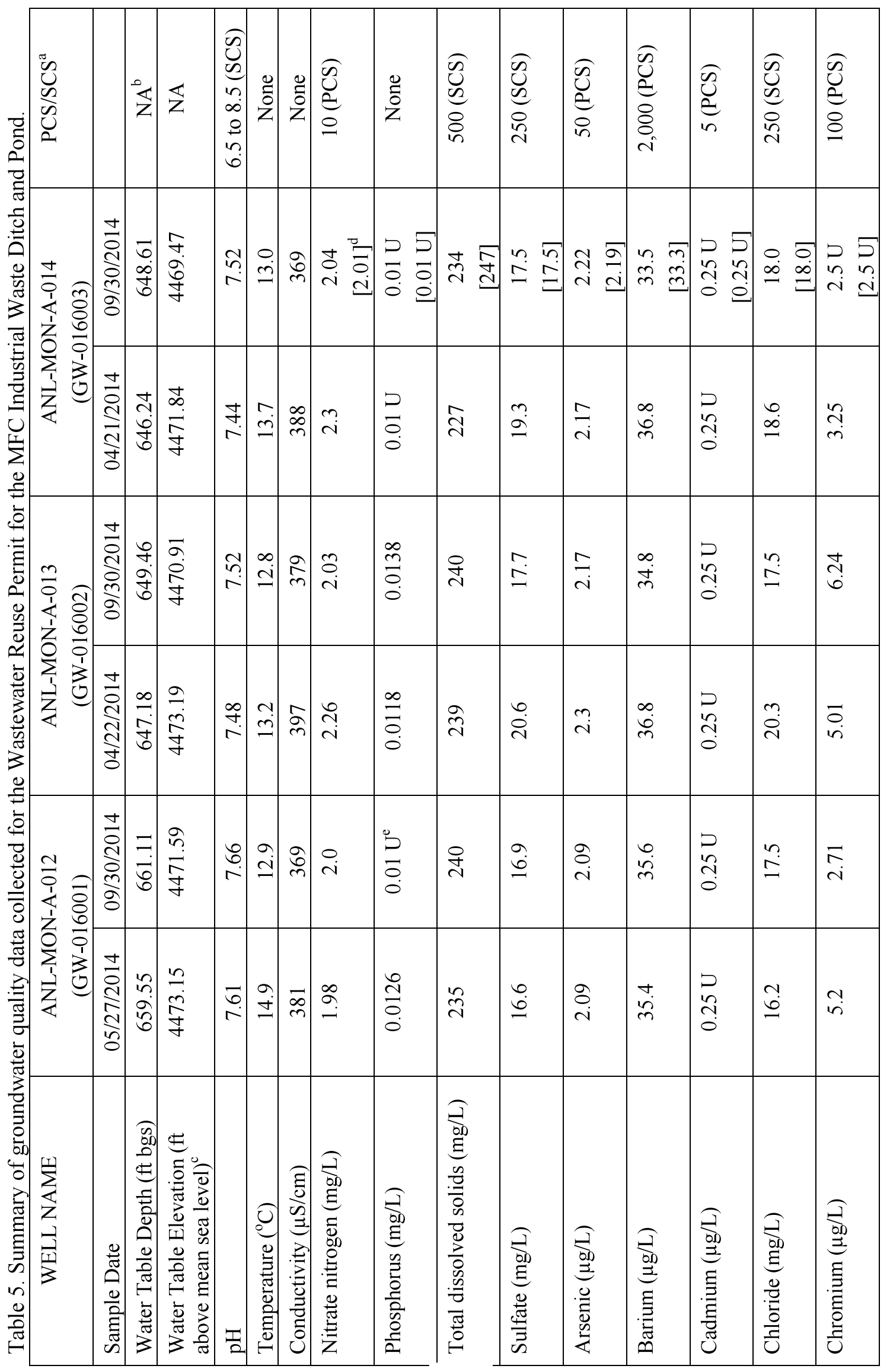




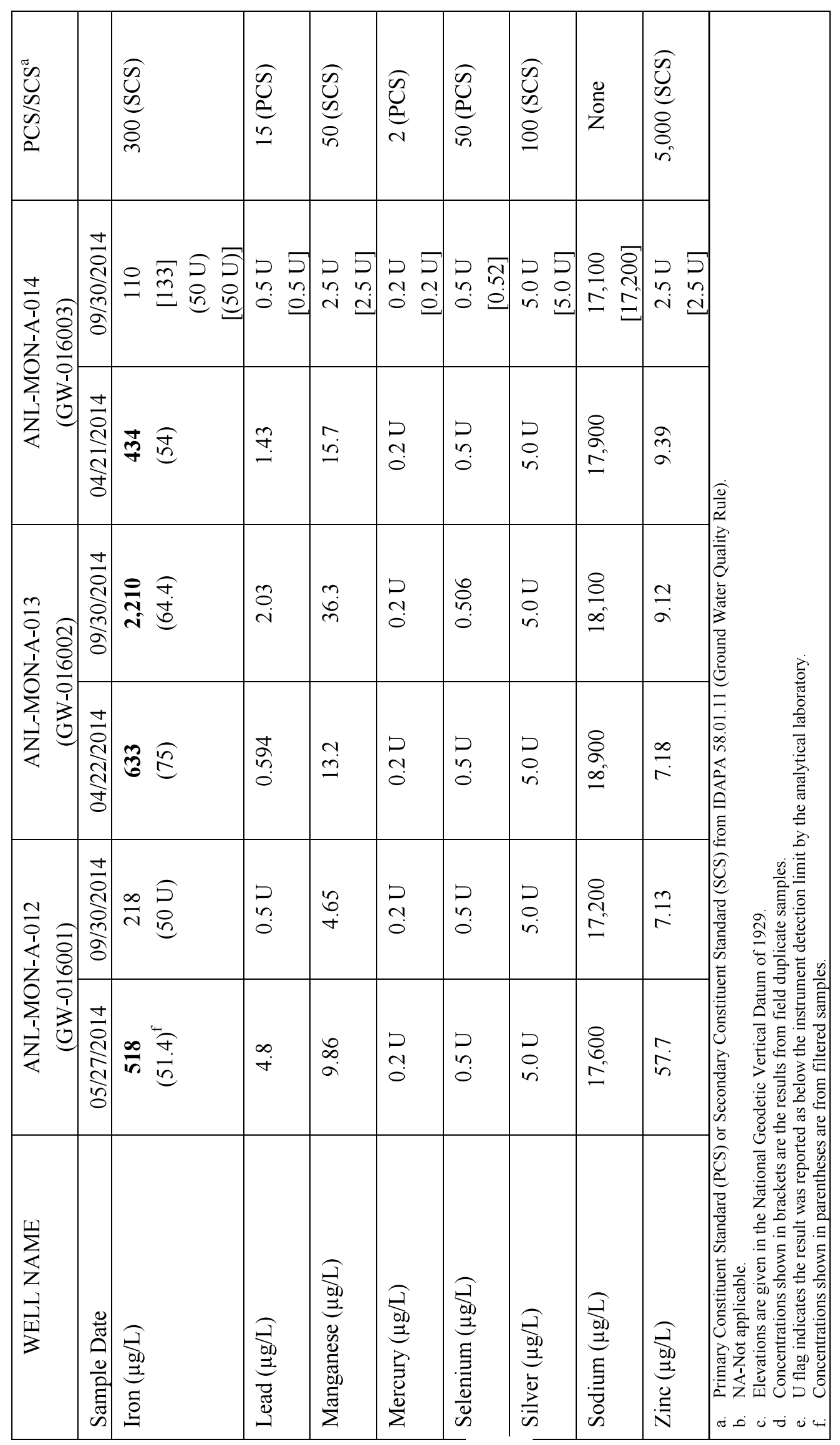




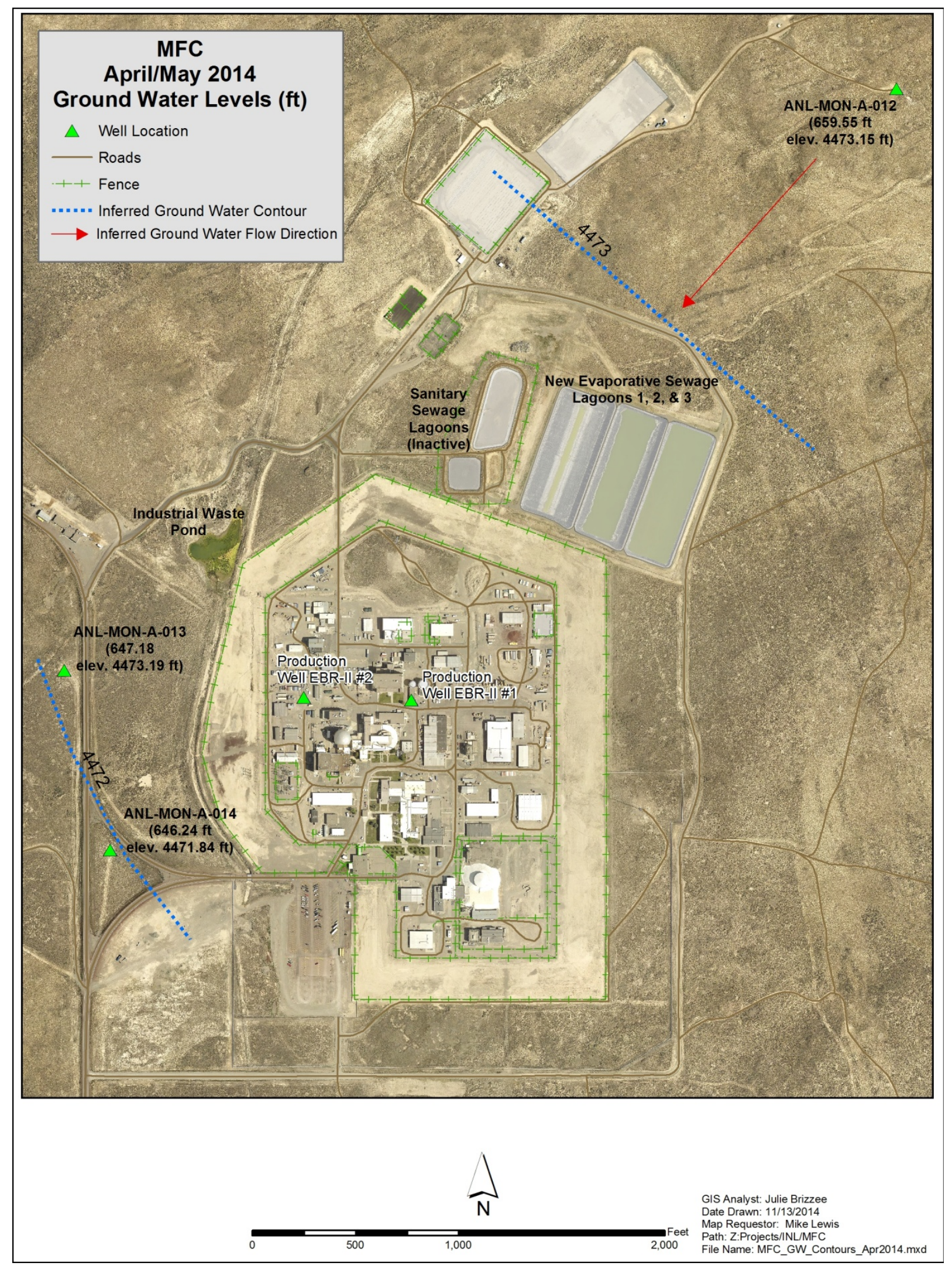

Figure 4. Groundwater contour map based on the April/May 2014 water level measurements. 




Figure 5. Groundwater contour map based on the September 2014 water level measurements. 


\section{PERMIT YEAR SUMMARIES}

This section provides information and status associated with permit required compliance activities. Noncompliance issues are also addressed in this section.

\subsection{Status of Permit Required Compliance Activities}

Section E of the IWRP identifies two compliance activities: preparation of a Plan of Operation and a Waste Solids Management Plan. Section H, Paragraph 5, of the permit requires that DEQ be notified within 30 days of completing any work described in Section E, and that the annual report shall provide the status of compliance activities still in progress at the end of the permit year.

Compliance Activity CA-160-01: This compliance activity to submit a final Operation and Maintenance Manual was completed in June 2011 with the approval letter from the DEQ (Rackow 2011).

Compliance Activity CA-160-02: This compliance activity requires a Waste Solids Management Plan shall be submitted to DEQ as needed. The compliance activity states:

"A Waste Solids Management Plan shall be submitted for DEQ review and shall be approved by DEQ prior to any dredging or removal of solids, mud, or sludge from the Industrial Waste Pond. The plan shall outline actions associated with the removal (dredging) of solids in the Industrial Waste Pond. The plan shall include: specific information used in the determining the need for removal of solids, responsible person(s) for the decision, and a complete SOP for the removal of the solids."

The due date for CA-160-02 is as needed, review and approval required prior to removal of any waste solids. Currently there are not any plans to remove solids from the pond.

\subsection{Noncompliance Issues}

The second quarter (June 11, 2014) TSS sample result from Ditch C was $182 \mathrm{mg} / \mathrm{L}$. This sample result exceeded the permit requirement of $100 \mathrm{mg} / \mathrm{L}$. In accordance with the IWRP, a letter (Miller 2014c) identifying the exceedance was submitted to DEQ.

On July 28, 2014, a follow up TSS sample was collected. The TSS concentration in the July sample was $6 \mathrm{mg} / \mathrm{L}$. TSS concentrations for the other three quarterly samples (Table 2) were below the laboratory instrument minimum detection level of $4 \mathrm{mg} / \mathrm{L}$. Typically, TSS concentrations from this location are below the laboratory instruments minimum detection level (Figure 6).

A review of the operations that contribute to the Ditch $\mathrm{C}$ discharge did not reveal any changes that would have caused the high TSS. Quarterly sampling will continue in accordance with the IWRP. 


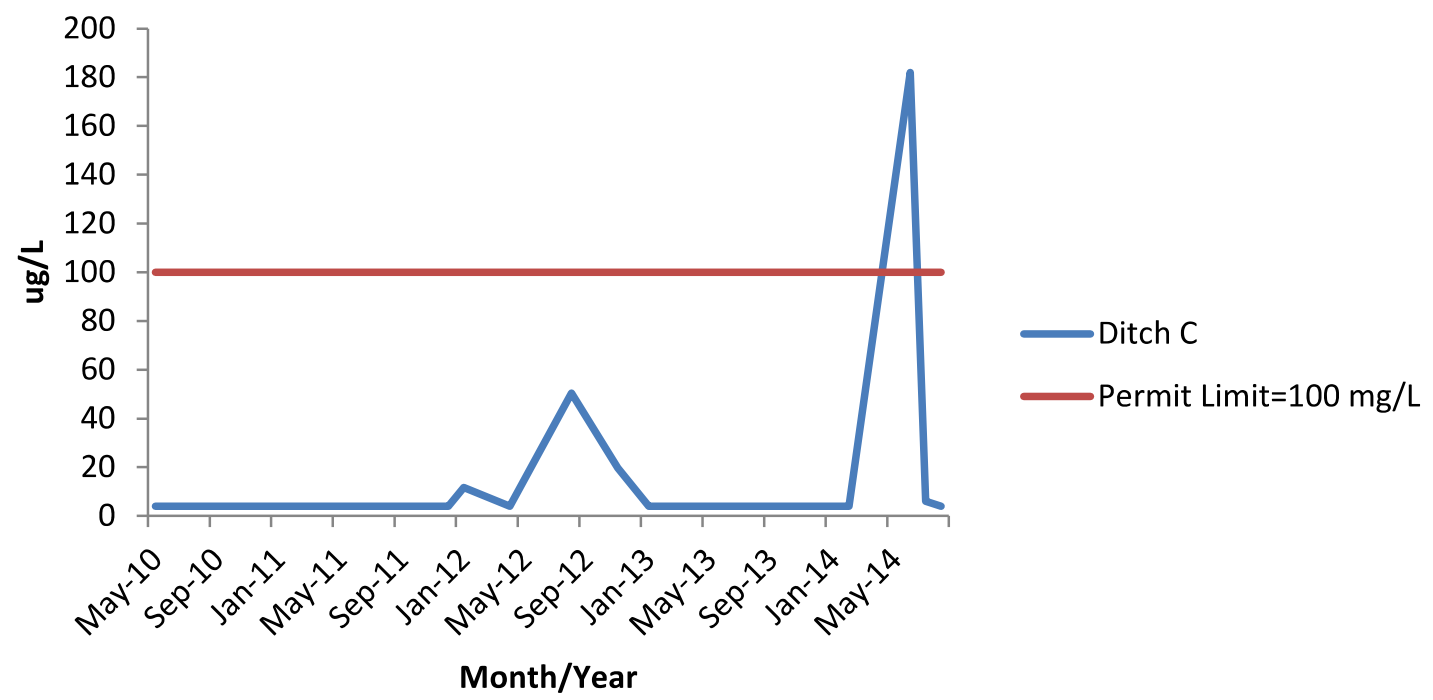

Figure 6. Total suspended solids concentrations in samples collected from Ditch C for the period of May 2010 through August 2014.

\section{ENVIRONMENTAL IMPACTS}

The IWRP specifies a maximum hydraulic loading rate of $17 \mathrm{MG} / \mathrm{year}$. The total volume discharged to the MFC Industrial Waste Ditch and Pond during the reporting period (November 1, 2013 through October 31, 2014) was $10.11 \mathrm{MG}$.

With the exception of a total suspended solids (TSS) sample collected on June 11, 2014, from the Industrial Waste Water Underground Pipe (Ditch C), the effluent from the Industrial Waste Pipeline and the Ditch $\mathrm{C}$ met the permit limits, based on a 30-day average, for total nitrogen (TN) and TSS of $20 \mathrm{mg} / \mathrm{L}$ and $100 \mathrm{mg} / \mathrm{L}$, respectively. No permit limits are specified for the other analytes.

The second quarter (June 11, 2014) TSS sample result from Ditch C was $182 \mathrm{mg} / \mathrm{L}$ and exceeded the permit requirement of $100 \mathrm{mg} / \mathrm{L}$. A follow up sample collected on July 28, 2014 had a concentration of 6 $\mathrm{mg} / \mathrm{L}$. TSS concentrations for the other three quarterly samples (Table 2) were below the laboratory instrument's minimum detection level of $4 \mathrm{mg} / \mathrm{L}$. Typically, TSS concentrations from this location are below the laboratory instrument's minimum detection level. There are no expected negative impacts to Ditch C.

The concentrations of the permit-required analytes in the groundwater samples were all below the respective groundwater quality standards in IDAPA 58.01.11 as required by the IWRP. Filtered results for iron were used to show compliance when the unfiltered sample result exceeded the groundwater quality standard.

The concentrations of chloride, nitrate+nitrite (as nitrogen), phosphorus, and sodium appear to be elevated in the effluent from the Industrial Waste Pipeline and Ditch C; however, the concentrations of these constituents in the down gradient monitoring wells are nearly indistinguishable from concentrations in the up gradient well.

There are positive impacts to the environment associated with the operation of the Industrial Waste Pond. These include aquifer recharge and providing a needed source of water for numerous species of native wildlife. 


\section{REFERENCES}

40 CFR 136, 2014, "Guidelines Establishing Test Procedures for the Analysis of Pollutants," Code of Federal Regulations, Office of the Federal Register, July 2014.

40 CFR 141, 2014, "National Primary Drinking Water Regulations," Code of Federal Regulations, Office of the Federal Register, July 2014.

40 CFR 143, 2014, "National Secondary Drinking Water Regulations," Code of Federal Regulations, Office of the Federal Register, July 2014.

IDAPA 58.01.11, 2009, "Ground Water Quality Rule," Idaho Department of Environmental Quality, July 2009.

Miller, T. A., INL, to E. Neher, DEQ, October 28, 2014a, “Transmittal of Idaho National Laboratory Recycled Water Reuse Permit Renewal Application for the Materials and Fuels Complex Industrial Waste Ditch and Industrial Waste Pond and Application Forms," CCN 234270.

Miller, T. A., INL, to T. Rackow, P. E., DEQ, September 23, 2014b, "Idaho National Laboratory Materials and Fuels Complex Industrial Waste Pond Wastewater Reuse Permit (WRU-I-0160-01), Update of Associated Groundwater Monitoring Well Diagrams for Wells GW-016001, GW-016002, and GW016003," CCN 233981.

Miller, T. A., INL, to G. Eager, P. E., DEQ, August 14, 2014c, "Idaho National Laboratory Materials and Fuels Complex Industrial Waste Pond Wastewater Reuse Permit (WRU-I-0160-01), Exceedance of the 100 mg/L Permit Limit for Total Suspended Solids," CCN 233768.

Neher, E., DEQ, to W. F. Hamel, DOE-ID, and D. Coburn, BEA, April 14, 2010, "Materials and Fuels Complex (MFC) Industrial Waste Ditch (IWD) and Industrial Waste Pond (IWP), Industrial Wastewater Reuse Permit No. LA-000160-01," CCN 220726.

Neher, E., DEQ, to W. F. Hamel, DOE-ID, and C. Melbihess, BEA, June 21, 2012, "Materials and Fuels Complex (MFC) Industrial Waste Ditch (IWD) and Industrial Waste Pond (IWP), Industrial Wastewater Reuse Permit No. WRU-I-0160-01 (formerly LA-000160-01), Modification 1," CCN 227704

Rackow, T, DEQ, to J.A. Stenzel, INL, June 23, 2011, "LA-000160-01 INL MFC Industrial Wastewater Pond, CA-141-03 Plan of Operation Approval," CCN 224615. 
Radiological Monitoring Results for Groundwater Samples Associated with the Industrial Wastewater Reuse Permit for the Materials and Fuels Complex Industrial Waste Ditch and Pond: November 1, 2013October 31, 2014 



\begin{abstract}
This report summarizes radiological monitoring performed on samples from specific groundwater monitoring wells associated with the Industrial Wastewater Reuse Permit for the Materials and Fuels Complex Industrial Waste Ditch and Industrial Waste Pond WRU-I-0160-01, Modification 1 (formerly LA-00016001). The radiological monitoring was performed to fulfill Department of Energy requirements under the Atomic Energy Act.
\end{abstract}




\section{CONTENTS}

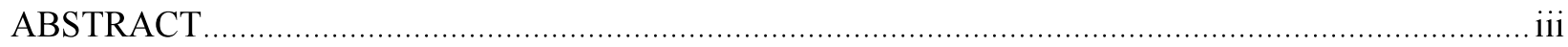

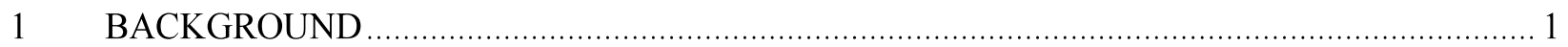

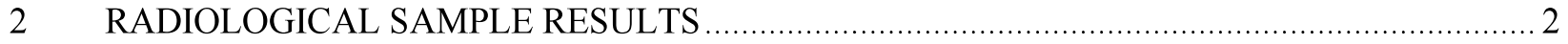

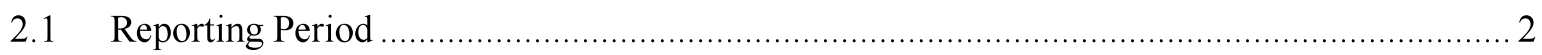

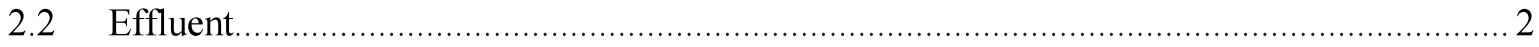

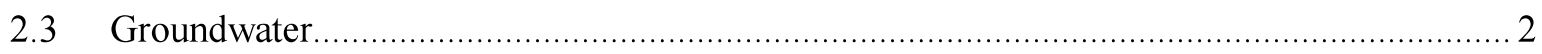

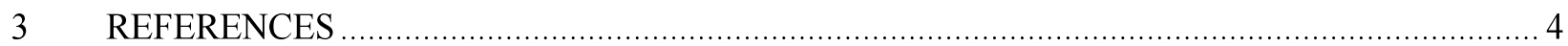

TABLES

Table 1. Positive detections in groundwater samples collected in 2014 from the monitoring wells sampled for the MFC Industrial Waste Ditch and Pond IWRP. 


\section{Radiological Monitoring Results for Groundwater Samples Associated with the Industrial Wastewater Reuse Permit for the Materials and Fuels Complex Industrial Waste Ditch and Pond: November 1, 2013- October 31, 2014}

\section{BACKGROUND}

The radiological information presented in this report is provided based upon an agreement between the Idaho Department of Environmental Quality and the U.S. Department of Energy Idaho Operations Office. The agreement is documented in the Industrial Wastewater Reuse Permit (IWRP) WRU-I-016001 (formerly LA-000160-01), Modification 1, for the Idaho National Laboratory Site's Materials and Fuels Complex (MFC) Industrial Waste Ditch (IWD) and Industrial Waste Pond (IWP). The initial IWRP was issued in April 2010 (Neher 2010). Modification 1 was issued in June 2012 (Neher 2012). Items 7 and 8 of Section H ("Standard Reporting Requirements") of the IWRP state:

- The permittee agrees to provide to the Department the results of ground water radiological monitoring with respect to the MFC Industrial Waste Pond and Ditch hydraulic management unit (HMU) that is performed to fulfill Department of Energy requirements under the Atomic Energy Act. The permittee agrees to provide the results with the Annual Report.

- The permittee agrees to provide to the Department the results of radiological monitoring of the MFC effluent, prior to discharge into the HMU, with respect to the MFC Industrial Waste Pond and Ditch HMU that is performed to fulfill Department of Energy requirements under the Atomic Energy Act. 


\section{RADIOLOGICAL SAMPLE RESULTS \\ 2.1 Reporting Period}

For the MFC Industrial Waste Pond and Ditch, the 2014 reporting year runs from November 1, 2013 through October 31, 2014. As stated in the "Facility Monitoring Table" of Section G of the IWRP, groundwater sampling shall be conducted in April/May and September/October.

\subsection{Effluent}

Composite samples were collected monthly from the Industrial Waste Pipeline (WW-016001) over a 24-hour period. Grab samples were collected quarterly from the wastewater discharged to Ditch C from the Industrial Waste Water Underground Pipe (WW-016002). No radiological analyses are performed on samples collected at these locations.

\subsection{Groundwater}

Groundwater samples for radiological parameters were collected from aquifer wells ANL-MON-A012, ANL-MON-A-013, ANL-MON-A-014 in April, May (ANL-MON-A-012) and September 2014. The samples were shipped under full chain of custody to GEL Laboratories in Charleston, South Carolina, and analyzed for gamma spectrometry, gross alpha, gross beta, tritium, and alpha spectroscopy. Samples were collected to satisfy the release objectives of DOE Order 458.1 addressed within the Environmental Management System as required by DOE Order 436.1, and requirements for the Comprehensive Environmental Response, Compensation, and Liability Act.

Table 1 shows the positive detections in groundwater samples. Positive detections are considered measurements exceeding the instrument's minimum detection level and greater than two times the uncertainty. Alpha/beta activity, uranium-234, and uranium-238 were detected in both the upgradient well (ANL-MON-A-012) and the downgradient wells (ANL-MON-A-013 and ANL-MON-A-014) at low levels. Alpha/beta activity, uranium-234, and uranium-238 all occur naturally and are not necessarily indicative of anthropogenic impacts. 
Table 1. Positive detections in groundwater samples collected in 2014 from the monitoring wells sampled for the MFC Industrial Waste Ditch and Pond IWRP.

\begin{tabular}{|c|c|c|c|}
\hline Monitoring Well & Sample Date & Parameter & $\begin{array}{c}\text { Sample Result } \\
(\mathrm{pCi} / \mathrm{L})\end{array}$ \\
\hline \multirow{7}{*}{$\begin{array}{c}\text { ANL-MON-A-012 } \\
\text { (upgradient) }\end{array}$} & $05 / 27 / 14$ & Gross alpha & $1.52( \pm 0.384)^{\mathrm{a}}$ \\
\hline & & Gross beta & $3.11( \pm 0.591)$ \\
\hline & & Uranium-233/234 & $1.09( \pm 0.172)$ \\
\hline & & Uranium-238 & $0.656( \pm 0.126)$ \\
\hline & $09 / 30 / 14$ & Gross beta & $4.43( \pm 0.761)$ \\
\hline & & Uranium-233/234 & $1.48( \pm 0.178)$ \\
\hline & & Uranium-238 & $0.573( \pm 0.0954)$ \\
\hline \multirow{7}{*}{$\begin{array}{c}\text { ANL-MON-A-013 } \\
\text { (downgradient) }\end{array}$} & $04 / 22 / 14$ & Gross alpha & $2.12(0.745)$ \\
\hline & & Gross beta & $4.94( \pm 1.09)$ \\
\hline & & Uranium-233/234 & $1.42( \pm 0.203)$ \\
\hline & & Uranium-238 & $0.693( \pm 0.13)$ \\
\hline & $09 / 30 / 14$ & Gross beta & $3.17( \pm 0.848)$ \\
\hline & & Uranium-233/234 & $1.28( \pm 0.153)$ \\
\hline & & Uranium-238 & $0.578( \pm 0.0911)$ \\
\hline \multirow{10}{*}{$\begin{array}{c}\text { ANL-MON-A-014 } \\
\text { (downgradient) }\end{array}$} & $04 / 21 / 14$ & Gross alpha & $3.4( \pm 1.06)$ \\
\hline & & Gross beta & $5.6( \pm 0.971)$ \\
\hline & & Uranium-233/234 & $1.21( \pm 0.205)$ \\
\hline & & Uranium-238 & $0.806( \pm 0.159)$ \\
\hline & 09/30/14 & Gross beta & $4.17( \pm 0.964)$ \\
\hline & & & $5.14( \pm 0.891)^{b}$ \\
\hline & & Uranium-233/234 & $1.49( \pm 0.183)$ \\
\hline & & & $1.32( \pm 0.171)^{b}$ \\
\hline & & Uranium-238 & $0.615( \pm 0.103)$ \\
\hline & & & $0.553( \pm 0.0971)^{b}$ \\
\hline
\end{tabular}




\section{REFERENCES}

42 USC § 2011-2259, 1954, “Atomic Energy Act of 1954," United States Code.

DOE Order 436.1, 2011, "Departmental Sustainability," U.S. Department of Energy, May 2, 2011.

DOE Order 458.1, 2011, "Radiation Protection of the Public and the Environment," U.S. Department of Energy, February 11, 2011.

Neher, E., DEQ, to W. F. Hamel, DOE-ID, April 14, 2010, "Materials and Fuels Complex (MFC) Industrial Waste Ditch (IWD) and Industrial Waste Pond (IWP), Industrial Wastewater Reuse Permit No. LA-000160-01," CCN 220726.

Neher, E., DEQ, to W. F. Hamel, DOE-ID, June 21, 2012, "Materials and Fuels Complex (MFC) Industrial Waste Ditch (IWD) and Industrial Waste Pond (IWP), Industrial Wastewater Reuse Permit No. WRU-I-0160-01 (formerly LA-000160-01), Modification 1," CCN 227704. 